\title{
The Centrencephalic Space of Functional Integration: a Model for Complex Intelligent Systems
}

\section{Nelson Mauro Maldonato ${ }^{1}$, Raffaele Sperandeo ${ }^{2}$, Paolo Valerio ${ }^{1}$, Marzia Duval $^{1}$, Cristiano Scandurra ${ }^{1}$, Silvia Dell'Orco ${ }^{3}$}

\author{
${ }^{1}$ University of Naples Federico II, Department of Neuroscience and Reproductive \\ and Odontostomatological Sciences, Via Sergio Pansini, 5, 80131 Naples, Italy, \\ nelsonmauro.maldonato@unina.it,paolo.valerio@unina.it, \\ marzia.duval@unina.it, cristiano.scandurra@unina.it \\ ${ }^{2}$ University of Basilicata, Department of Human Sciences, Via N.Sauro 85, 85100 \\ Potenza, Italy, raffaele.sperandeo@unibas.it \\ ${ }^{3}$ University of Naples Federico II, Department of Humanistic Studies, Via Porta di \\ Massa, 1, 80133 Naples, Italy, silvia.dellorco@unina.it
}

\begin{abstract}
If we have recently begun to understand how DNA gives life to embryos and then to individuals, only very little is understood of the intricate interactions between the biological bases of life, the environment and the human brain. The exponential acceleration of technological change could change many, perhaps all, the rules that have guided our civilization so far. It is very likely that these intelligent artificial entities will take much less time to understand the codes that constitute them, gaining forms of (self) awareness, decision-making skills, introspective capacities, mind reading and even free will. If all this is achieved, in the coming decades humanity will be destined to a profound cultural, epistemological and even physiological transformation. In this paper, we aim to show how the success or failure of a balanced man-machine co-evolution will also depend on some answers to fundamental scientific questions that have remained unexplored, such as consciousness and decision-making, creativity, but above all to the adaptive factor that more radically sustained and pushed the evolution beyond the constraints of our genetic code: improvisation. This entanglement of neuronal matrices could be at the origin of an intermodal communication consists of a stream of semantic phenomena, mental images and more, tuned thanks to "pattern recognition" in centrencephalic space of functional integration - thus explaining "remote spectrum actions" at the base of primary adaptive unconscious and experiences life.
\end{abstract}

Keywords: consciousness; decision-making; creativity; improvisation; intelligent systems communication 


\section{Introduction}

Technological progress is not just one of the most distinctive signs of social organization, but a crucial propulsive factor in human evolution. More than the outcome of rigid selective mechanisms, the technological process has so far represented a co-evolutionary process [1]. Morever, if human evolution has been marked by the intervention of natural variables, technological evolution has depended on an artificial selection made by man. Today, the role of technology appears ever more pervasive and powerful, in social life and in individual life. In fact, there are more and more people who believe that this will generate, sooner or later, organisms capable of going far beyond the simulation of human brain functions: that is, hybrids that will learn from their internal states, interpret reality data, establish their own objectives, will converse with humans; above all, they will decide based on their own 'value system' [2]. In a not too distant future, these organisms could acquire ever wider spheres of autonomy, self-conservative instances, hierarchies of values, perhaps an ethic based on 'freedom' [3]. Of course, it will be difficult to redo the immense work of evolution: for example, calling experience an elaboration (even if sophisticated) of information or emotions such as pain or pleasure [4]. However, we can really exclude that one day these entities will have no spirit of initiative and capacity for discernment? At least on the theoretical level, there are no logical obstacles that can exclude that one day this could happen even with thinking machines [5]. Our current inability to answer this question must urge us to look at things from unfamiliar perspectives.

\section{Consciousness, Decision-Making, Creativity, Improvisation: the Unavoidable Questions}

Despite the enormous progress made in the field of neuroscience and AI, the discussion on the possibility of constructing an artificial consciousness still clashes with the dramatic lack of scientific notions on the functioning of biological consciousness [6]. The same term 'consciousness' continues to be used as a sort of passe-partout to indicate different and distant phenomena: the coma, the vegetative state, the environmental sensitivity, the moral, the activity of the ego and so on [7]. After a centuries-old terminological confusion, we must ask ourselves if it is not time to demarcate this fundamental research object more rigorously on the scientific and semantic level. For a long time scholars have argued that basic characteristics of consciousness were unity and permanence over time. On the other hand, several studies show that it represents a multiple process that contains, simultaneously, distinct contents, each of which with its own intentionality [8]. What are the underlying biophysical mechanisms? And how does this multiplicity express itself unitarily in its different states and contents? Schematizing there are two possible models. In the first model, to generate consciousness would be a 
central neural system, in which duly integrated information is first brought to representation and then allowed to emerge in awareness. According to this representation, consciousness appears as the expression of an elaboration of the corticosubcortical system that generates different contents and representations, accomplishing exclusively in the brain [9]. In the second model, the simultaneous coactivation of content generated by structures distributed in the brain would ultimately result in the phenomenon of awareness. Consciousness would thus be generated by brain mechanisms distributed in the brain - both cortical and subcortical - whose contents, independent of each other, are exposed to intrasensorial and intersensory (environmental) influences that, influencing each other, co-determine the 'conscious experience'. It is from here that the distinction between a unitary model and a plural model of consciousness passes [10].

How can multiple neural events give us the impression of a unitary subjectivity? And what are the steps towards the constitution of the Self and of awareness? Of course, concepts such as "subjectivity" and "self" still remain problematic. Here, the Self is understood as an emerging phenomenon when the individual events produced by the brain are sufficiently representative, coherent and cohesive. We experience a structured world of distinct and ordered objects in space, organized according to regularity and content within significant spatio-temporal patterns: extramodal contents (colours, form, etc.) and intramodal (proprioceptive, auditory and visual, etc). Representative cohesion is not an invariant characteristic of conscious experience, but the result of a selection by which the brain seeks the path of its own integration [11]. Thus, the Self has to do with an ordering activity of the conscience, which elaborates and sustains this multiplicity in an interweaving of local contents in relation to one another [12]. In such a model, consciousness appears no longer as a hierarchical structure, but a multiple horizontal entity, whose representative cohesion is operated by distributed thalamo-cortical and corticocortical circuits [13]. All conscious experiences, starting with qualitative experiences, are unified within the field of consciousness. Unity, therefore, is implicit in qualitative subjectivity. In other words, if our awareness is determined by the play of these innumerable dynamics, then there are only different unified states of consciousness in subjectivity, as well as aggregate underlying fields of consciousness [14]. As is evident, the question of conscious subjectivity goes beyond the search for its neural correlates and beyond the contraposition between consciousness and the unconscious. In the phenomenon of vision, for example, the relevant question certainly concerns the neural correlates of consciousness, but above all the way in which visual experiences become part of awareness. If the architecture of the field of consciousness is the thalamo-cortical system — which elaborates information from different districts in different sensory modalities (visual, tactile, auditory, etc.) - from its neural operational levels one could trace the structure of consciousness visual, qualia, temporal experience and more [15]. Whatever the case, the brain cannot generate a conscious experience by itself. In fact, it is only a necessary condition because innumerable neuronal micro-events generate conscious perceptions of the objects of the world [16]. The study of consciousness 
requires multilevel research criteria: a quantitative-categorical (attention, vigilance, sleep, and coma); a qualitative-dimensional (subjective experiences such as feelings, thoughts, emotions); one, finally, for the analysis of the different types and degrees of synchronic consciousness (the field of consciousness) and diachronic (the ego or personality). In the field of the Artificial Consciousness (AC) and the Artificial General Intelligence (AGI) [17] the decisional processes and the conscience require the integration of processes and inputs coming from different sources. The creation of an artificial conscience, however, brings with it a series of ethical questions to which we would not be ready to respond. For example, machines with consciousness could experience emotions, empathy, and free will? In this last case, could they emancipate themselves from their condition? To date, no one has yet managed to create an artificially conscious entity. It is therefore; difficult to say what characteristics it will have [18]. At the state, even if unconscious, artificial agents can make decisions in many situations (which naturally becomes a central factor in complex environments) by applying normative rules to the information available. Think about situations that require reflection and implementation of moral principles, the ability to recognize and deal with ethically significant situations, the ability to discriminate essential information from irrelevant information, the search for new data in the event of insufficient information, judgments on the intentionality of other agents with whom it interacts, to choose a course of action that minimizes the damage [19]. All of this, of course, within the time constraints required by the situation.

The creation of artificial agents capable of making moral decisions is among the most difficult challenges faced by the AI. In recent decades, a growing body of research has shown that much of the moral behavior derives from unconscious judgments. It is clear that if on the one hand these results do not exclude the presence of logical-formal, conscious, and deliberative thinking. Howerver, they underline how conscious reflection is less frequent than imagined. For example, the Social Intuitionist Model [20] underlines - in the context of moral decisions as well as in simpler situations - the primacy of intuition on conscious reasoning. In reality, starting from the second half of the twentieth century, the model of rational agent has gradually lost credibility $[21 ; 22 ; 23 ; 24]$, restoring centrality to factors such as unpredictability and uncertainty. The analysis of real-world behaviors has shown that we often decide using simplified schemes, distorted representations and perceptions, extra-cognitive factors such as emotional assessment of situations, fear of the consequences of an action, tolerance to frustrations, courage, creativity. Not to mention the risk situations, in which we often rely on partial or insufficient information deriving from past and present experiences, prejudices, conjectures [25]. In other words, for most of the time, the mind works with instruments other than formal logical ones. It is not surprising, therefore, that despite, the incredible advances in AI and robotics there are still no artificial agents capable of improvising and making adaptive decisions [26]. Developing an artificial agent capable of adapting and deciding autonomously according to these spheres of 'natural logic' would mean reproducing reasoning based, as in man, on 
unconscious and non-deductive inferences $[27 ; 28]$. In this sense, the horizon of logic, both human and artificial, is much broader than that of traditionally understood formal logic.

\section{Improvisation and Centrencephalic Space for Functional Integration}

The study of improvisation helps us to understand not only the relationship between conscious and unconscious actions, the complex neural basis of executive functions, and more: basic categories of human action [29]. Until now, the prevailing theoretical models have focused their attention above all on the correlates between the cortical areas and the related cognitive processes. Little attention, however, has been assigned to the great variety of subcortical activities, in particular those of the basal ganglia: fundamental subcortical structure, whose implicit procedures and the role played in the memory processes generate continuous novelties that allow the prefrontal cortex to transform a huge and untidy amount of information in explicit creative behavior [30]. The basal ganglia are, moreover, strongly involved in the activation of the chemical signals generated by dissonances or asymmetries between perceptions and expectations, intervening according to the circumstances also in the responses to the environmental needs. In this sense, they interact with the frontal cortex and the limbic system, exercising a key function in planning, in selecting appropriate actions and in motor decisionmaking processes [28]. For a long time the ability to improvise was understood as a skill acquired only after a long and intense practice. Although necessary, this precondition appears to be insufficient [31]. In fact, there are other factors that play a crucial role in improvisation: the time constraints, the simultaneity of senseperceptual coding, performance monitoring and more [32]. Moreover, it has long insisted on the importance, in the improvisation, of the interaction between perceptive-emotional processes and specific knowledge that, once recovered, would minimize the processing processes favoring the generation of original ideas [33]. In this scheme, an essential role would be played by error correction feedback systems that would ensure such fluidity to improvisation, through automated processes that require the least amount of conscious attention. In this way, the space for new action sequences would be reserved for the lower levels of motor control, while the correction of errors would proceed from a higher order to a lower order and individual goal directed movements would be combined in routine and subroutine sequences. Years ago, Schmidt [34] hypothesized the existence of a generalized program ("motor scheme theory") that would select flexible motion patterns for modulation of action in context changes. Subsequently, other theories have suggested the existence of 'unity of action' system, of 'organizational invariants' [35], is open, flexible models, far from equilibrium and adaptable to critical fields different. These are models that, applied to motor action, would leave neuromus- 
cular properties and patterns of coordination emerging from the constraints imposed by task situations, giving life, from time to time, to solutions for specific environmental problems.

\section{Generative Structures}

Experimental evidence suggests a correlation between improvisation and divergent thinking [36], cognitive flexibility, the widespread activation of semantic networks [37] and a structural organization of semantic memory [38]. Central aspects in the research processes of creative cognition have been considered, on the one hand, the recovery of mnestic elements, the inhibition of the emerging response, the fluid intelligence, the working memory; and, on the other hand, the inhibition of potentially interfering information with the generativity of ideas [39]. Although in the literature on creativity there is no definitive evidence on a decisive role of divergent thinking and mental flexibility, different studies show how creative cognition involves brain regions connected with executive functions [30; 40]. Other studies show the constant activation of the left inferior frontal gyrus both in the generation of ideas and in the analysis of ideas recovered from longterm memory. In addition to the left inferior frontal gyrus, the improvisation activates the additional motor area, the left dorsolateral prefrontal cortex and, bilaterally, the insular cortex and the cerebellum. To stimulate the lower left frontal gyrus in the recovery of long-term memory data and to evaluate the neural correlates, [41], tasks requiring fluidity similar to those used to elicit verbal fluency were used in the assessment of executive deficits, which require control and strategic attention to access to memory [42]. In a recent meta-analysis of the literature on creative processes, a significant role was highlighted of the caudal and rostral prefrontal regions, as well as of the inferior and posterior parietal temporal areas. This network, which includes semantic regions related to the recovery and activation of remote mental representations, would allow the emergence of free generative activities. The significance of such evidence is also strengthened by a recent fMRI study [39] which reported, in individuals with high divergent thinking index, greater functional connectivity between the left lower frontal gyrus and the network default mode [39]. It seems, therefore, that the left lower frontal gyrus plays a crucial role in processes, such as musical improvisation, which require a controlled action of long-term memory [43]. It must be said, however, that while the generativity has been associated with a greater activation of the left lower frontal gyrus, the bilateral premotor cortex, the inferior and superior parietal lobes and the bilateral medial temporal lobes; for its part, the evaluation was associated with a greater activity of the network default mode in the control and in the execution [44]. Further analysis of functional connectivity during evaluation [39] highlighted a strong functional unity between executive and default networks, which indicates a greater cooperation between controlled and spontaneous thought processes. 


\section{Cognition and Improvisation}

But is there, and to what extent, a cognitive control in improvisation? If the deactivation of the dorsolateral prefrontal cortex plausibly supposes a suspension of conscious inhibitory monitoring, activation of the medial prefrontal cortex would suggest an activation independent of the network default mode [45], which can be associated with phenomena such as mind-wondering [46] that interrupt conscious control in favor of partial, or almost total, focusing of emerging spontaneous thought. This activity, in the absence of external task requests, highlights a functional connectivity model suggestive of an activation of internally directed attention. Such evidence would suggest that, in the course of improvisation, there may be a suppression of executive control and, conversely, an activation of regions related to the network default mode [45] — even if the activation of regions associated with executive functions always evokes a certain degree of cognitive control. In the improvisation, therefore, the deactivation of the temporoparietal junction - area located near the right angular turn and part of a network that includes the temporoparietal junction and the ventral frontal cortex - would reflect the top-down control during tasks that require focusing of the internal attention [47]. In this sense, if the activation of this network acts in an inhibitory way on the information not coherent with the current task [48], its deactivation could correspond to a focus of internally directed attention, as has been highlighted in the studies on the production of creative ideas [30], the elaboration of divergent thinking, creative writing and even in the invention of design products.

\section{Implicit and Explicit Processes}

This research would seem to credit the hypothesis according to which improvisation depends on the interaction between generative, executive and evaluative processes of new motor sequences, from performance monitoring, from facilitation of attentional processes to higher order objectives, from reduced processing needs to minimum and so on [49]. Now, despite this hypothesis admits the existence, in the improvisation, of a certain degree of ecological complexity, little role is recognized in the subcortical processes underlying these phenomena. In fact, a full integration of emotional, cognitive and motor information depends on the competition of two different systems [50]. The first, the explicit one, based on rules and conscious contents, is associated with the superior cognitive functions of the frontal and prefrontal lobes and of the medial temporal lobe; the second, the implicit one, more efficient and based on practical and not aware abilities, associated with abilities mainly supported by the basal ganglia [51]. In this representation, the explicit system supports a hierarchical processing of information in which most of the most sophisticated mental abilities depend on the higher order structure: the prefrontal cortex. Naturally, between these systems and the nervous structures from 
which these cognitive abilities depend, there is no rigid separation [52]. In fact, both systems can be activated in parallel and the striatum, fundamental structure of the basal ganglia, is also involved in explicit cognitive functions due to the complex connections that associate it with the prefrontal cortex [53]. Furthermore, the striatum combines information from different cortical areas for the convergence of their respective terminal fields. Evidence on the function of the ventral striatum shows how the accumbens exercises not only a central role in positively or negatively reinforced behaviors, but also represents a crucial junction of the emotional information processing network constituted by the amygdala, the mesencephalic centers, the hippocampus and from the prefrontal cortex [54]. This complex network explains its centrality in the elaboration and conversion of information into suitable pipelines, which can possibly be reinforced. Beyond that, the ventral striatum anticipates the gratifications of the choices and signals the negative outcomes of the expected behaviors as reward [55]. Studies on reward systems have also highlighted the existence of an anticipation of reward. In fact, for the connection between the parts and the whole in the perception of a piece, the attack of a composition - for example a melodic fragment or the beginning of a musical phrase - creates an expectation of completion of the composition or sense of the sentence [56]. When this completion does not take place, or occurs incongruously, a particular wave appears in the EEG: the wave N 400 [57]. Furthermore, it has been suggested that stimuli detected in new or unexpected contexts activate the basal ganglia that verify the reliability of the predictions formulated in the prefrontal cortex [58]. Expectations may be cognitive or motor and it is reasonable to assume that the basal ganglia are massively involved in the activation of chemical signals evoked by dissonances or discrepancies between perceptions and expectations. Furthermore, depending on the needs of the moment, the ventral striatum also intervenes in the adaptation of cognitive strategies to environmental needs. This is how the subcortical reinforcement mechanisms, through their interaction with the frontal cortex and the limbic and striatal systems, play a key role in planning, selecting appropriate actions and decision-making processes [59]. As is known, the primary processes of thought - free associations, mind wondering, daydreaming, etc. from which analogies and creative ideas often emerge - take place at intermediate levels of activation, while secondary processes (characterized by an abstract, logical and reality-oriented cognition) require attention and have higher levels of activation [52]. It has been suggested that activation of the prefrontal cortex blocks 'irrelevant' behaviors and mental associations, while increasing target oriented behavior [60]. The continuum primary process-secondary process is the dimension along which cognition varies: in other words, creative individuals would, more than others, be able to oscillate between these two dimensions of thought, transiting towards a primary state of consciousness that would facilitate the discovery of new combinations of elements [61]. In this sense, the discovery of a solution (often improperly identified as creative behavior) is based on the ability to convert secondary processes into primary processes, thus leaving out analogies and free associations. Otherwise, the search for 
new solutions is linked to the ability to 'switch off' the prefrontal cortex and switch secondary processes into primary processes [62]. Years ago, Csikszentmihalyi [63] described an excited state of attention, related to a reduced prefrontal activity - called flow — in which the operations are almost automatic, without effort and concentration is so intense that it ignores all that it's around. This state plausibly calls implicit cognitive systems that allow the execution of tested skills and cognitive functions without interference of the explicit system [64]. In other words, it is a transitory state of minor activity of the prefrontal lobe, which temporarily 'off' the analytical abilities of the explicit system. Now, apparently the attention focused on the target seems to contradict the evidence of decreased frontal lobe activity previously reported. In fact, to be direct and persistent, the flow requires the activation of the frontal attentional network [65]. However, focused attention is also present in altered states of consciousness from transient hypofrontality. Furthermore, a flow state is compatible with a decrease in the prefrontal function that generates the attenuation of self-awareness [66]. For these reasons, flow is generally considered a lower state of frontal activity, with the exception of executive attention that allows the mind to focus on a target by 'shutting down' the other executive and cognitive abilities of the prefrontal cortex. Focusing on current activity allows the implicit system to be extremely efficient. Several studies show that the implicit striatal system reacts to novelty by generating new and consistent behaviors in response to environmental changes [67]. The prefrontal cortex is subsequently loaded, even if as soon as they are transformed into repetitive practices they are again managed by the basal ganglia, to be transformed into implicit procedures [68]. In this sense, the basal ganglia, with their implicit strategies and their memories, would constitute a mechanism that produces continuous novelties, while the prefrontal cortex (probably with its dorsolateral areas) transforms novelties into creative behaviors [30]. This is how the rich associative network - which allows the striatum to integrate motivational, cognitive and emotional information coming from different cortical areas and to transmit it to the prefrontal cortex - is a generative tool able to explain: a) the transformation of motor experiences and exploratory in cognitive schemes; b) the production of analogies at the base of creative discoveries. It is therefore, increasingly clear that every cognitive function depends on a multiplicity of components and not on a single structure or system [69]. Just as the language, which is not generated only by the motor and sensory areas of the left hemisphere, but also by the networks that connect these areas to the basal ganglia [70]. In this sense, if it is true that creativity must be considered starting from its neural and cognitive correlates: implicit and explicit strategies, the states of the primary and secondary mind, the executive abilities, the purpose-oriented behaviors, the emotions; it is equally true that a role no less decisive are the plastic processes that allow adaptation to the environment through new and original strategies. 


\section{Integrative Activities and Ordering Functions in the Functional Integration Space}

Historically, the insertion of improvisation within the cognitivist paradigm has placed secondary emphasis on cortico-subcortical interactions and unconscious motor activities [71]. Once again, a hierarchical representation of nerve functions has prevailed that leads to improvisation - asynchronous and simultaneous distributed activity of peripheral and central events - to the elaboration of information and contents, and to the cortical integration of specific domains and functions [72]. If it is clear that ordinarily our relationship life is marked by a flow of information and experiences ordered according to regularities and distinct contents within precise spatio-temporal patterns, in improvisation a simultaneous and sophisticated process of integration of extramodal and intramodal contents is realized, associated with fluid, coherent and coherent images and representations of harmonic and melodic materials, silences and sounds, executive abilities and expectations, possibilities and results [73].

However, through which modalities, and especially where, does this dialogue take place between neurons (and networks of neurons) that filters, selects, exchanges and coordinates information that will then be converted into perceptible actions and values? It is reasonable to believe that this functional integration takes place within a central-encephalic space [7], within which the prefrontal cortex 'dialogues' with the basal ganglia, integrating motivational, cognitive and emotional information coming from the thalamus and from other brain areas, for the transformation of sensorimotor and exploratory experiences into patterns and ideas at the highest level of abstraction [74]. This complex architecture includes, on the one hand, the prefrontal cortex, the parieto-temporal cortex and the cingulum gyrus; on the other, the orbito-frontal regions of the frontal cortex, which contribute to the subjective preferences of reward and pleasure; while the medial ones of the same frontal cortex intervene in the motivations and in the desire (the lateral parts of the latter intervene in the choices made according to the context, which is then the originality, the coherence, etc.). Without excluding, of course, a higher level: decision-making [75]. In fact, improvisation is an intense alternation of decisions.

The model of the space of centrencephalic functional integration is a sort of stable context-purpose, with ordering functions of the innumerable cortico-subcortical dynamics, which could explain transits without interruptions from one sensoryperceptive element to another. It would help us to reconsider the problematic awareness-unawareness, intentionality and ideo-motor activities at the highest level, as dimensional and non-discrete functions [76]. It would probably find its plausible explanation the same continuity of the links between rhythmic-melodic patterns, images, intuitions and everything that comes to it again, and which returns the sensation of duration [77]. In this space, fragments of memory merge with instant intuitions and current experiences, reverberating through interconnected political operations in others that are about to arrive. 


\section{Future Direction}

The questions presented in this paper are a prerequisite for understanding today some important aspects of our brain and our social life, before strong artificial intelligence changes the rules of the game. In fact, it seems evident that any change we have seen in the past will soon be abundantly overcome by the complexity of the implications of what we will see in the near future. To tackle the issues discussed here on the scientific level urges us to seek a more advanced experimental balance between ecological validity and control of mental functions: which means, then, not only modeling the understanding of our brain and our behavior, but also determining the different languages to describe it and draw alternative maps. Among those possible there is the "intermodal communication" concept for now inexplicable through the concept of linear causality between the different structures of our brains. This entanglement of neuronal matrices, which gives rise to a plot does not separable semantic phenomena, mental images, and more - tuned thanks to "pattern recognition" in centrencephalic space of functional integration - could explain the countless "remote spectrum actions" at the base of the adaptive unconscious and more general ones primary experiences of life. In this scheme the space itself may just be the device that gives us the illusion that things are far from each other: above all that the passage of information between different elements of a system can only occur through sequential, causal interactions to act spatially from start to finish. In terms of theoretical Neuroscience is move the observation, reflection and research at a different level, inscribing them inside values that they understand and incorporate the dynamics, experience and other 'forms of life' like those of complex intelligent systems. The wide spectrum of human behavior will be offset by the opportunity from the opportunity to experiment with more radical degrees of freedom. Understand this possibility if, on the one hand, means respecting those who want to preserve intact its human identity, the other allows whoever intends to explore what it means to be human in radically new ways and forms: choices, these, which will shape the society of the near future.

\section{References}

[1] Longo, G. O. (2003) Il simbionte: prove di umanità futura (Vol. 12) Meltemi Editore srl.

[2] Spence, S. (2009) The Actor's Brain: Exploring the Cognitive Neuroscience of Free Will. Oxford University Press

[3] Evers, K. (2009) Neuroéthique: quand la matière s'éveille. Odile Jacob

[4] Kurzweil, R. (2013) How to Create a Mind: The Secret of Human Thought Revealed. Penguin

[5] Poggio, T., Ullman, S. (2013) Vision: are Models of Object Recognition Catching up with the Brain? Annals of the New York Academy of Sciences, 1305(1), pp. 72-82 
[6] Dehaene, S., Lau, H., Kouider, S. (2017) What is Consciousness, and Could Machines Have It? Science, 358(6362), pp. 486-492

[7] Maldonato, N. M. (2009) From Neuron to Consciousness: For an Experience-based Neuroscience, World Futures, 65(2), pp. 80-93

[8] Zeki, S., Bartels, A. (1998) The Asynchrony of Consciousness. Proceedings of the Royal Society of London B: Biological Sciences, 265(1405), pp. 1583-1585

[9] Lamme, V. A. (2006) Towards a True Neural Stance on Consciousness, Trends in Cognitive Sciences, 10(11), pp. 494-501

[10] Kawamura, S. (1998) Multiple Streams of Time Consciousness: A New Model of Retrospective Timing. Perceptual and motor skills, 86(3), pp. $1119-1122$

[11] Tononi, G. (2004) An Information Integration Theory of Consciousness. BMC neuroscience, 5(1), p. 42

[12] Oizumi, M., Albantakis, L., Tononi, G. (2014) From the Phenomenology to the Mechanisms of Consciousness: Integrated Information Theory 3.0, PLoS computational biology, 10(5) pp. e1003588

[13] Damasio, A. R. (1998) Investigating the Biology of Consciousness. Philosophical Transactions of the Royal Society of London B: Biological Sciences, 353(1377) pp. 1879-1882

[14] Dehaene, S., Changeux, J. P. (2011) Experimental and Theoretical Approaches to Conscious Processing, Neuron, 70(2) pp. 200-227

[15] Baars, B. J. (1988) A Cognitive Theory of Consciousness. Cambridge University Press, New York

[16] Varela, F. J., Thompson, E., Rosch, E. (2017) The Embodied Mind: Cognitive Science and Human Experience. MIT press

[17] Wang, F. Y., Carley, K. M., Zeng, D., Mao, W. (2007) Social Computing: From Social Informatics to Social Intelligence, IEEE Intelligent Systems, 22(2)

[18] Lintas, A., Rovetta, S., Verschure, P. F., Villa, A. E. (Eds.) (2017) Artificial Neural Networks and Machine Learning-ICANN 2017: $26^{\text {th }}$ International Conference on Artificial Neural Networks, Alghero, Italy, September pp. 11-14, Proceedings (Vol. 10614) Springer

[19] McDermott, D. (2007) Artificial Intelligence and Consciousness. The Cambridge handbook of consciousness, 117-150

[20] Haidt, J. (2001) The Emotional Dog and Its Rational Tail: a Social Intuitionist Approach to Moral Judgment. Psychological review, 108(4), p. 814 
[21] Simon, H. A. (1955) A Behavioral Model of Rational Choice, The Quarterly Journal of Economics, 69(1), pp. 99-118

[22] Tversky, A., Kahneman, D. (1974) Judgment under Uncertainty: Heuristics and Biases, Science, 185(4157), pp. 1124-1131

[23] Maldonato, N. M., Dell'Orco, S. (2015) Making Decision under Uncertainty: Emotions, Risk and Biases, Smart Innovation, Systems and Technologies. Springer, Vol. 37, pp. 293-302

[24] Maldonato, N. M., Dell'Orco, S. (2011) How to Make Decisions in an Uncertain World: Heuristics, Biases, and Risk Perception, World Futures, 67(8), pp. 569-577

[25] Berthoz, A. (2006) Emotion and Reason: The Cognitive Neuroscience of Decision Making. OUP Catalogue

[26] Esposito, A., Esposito, A. M., Vogel, C. (2015) Needs and Challenges in Human Computer Interaction for Processing Social Emotional Information, Pattern Recognition Letters, 66, pp. 41-51

[27] Maldonato, N. M., Dell'Orco, S. (2013) The Natural Logic of Action, World Futures, 69, pp. 174-183

[28] Maldonato, N. M., Dell'Orco, S. (2010) Toward an Evolutionary Theory of Rationality. World Futures, 66(2), pp. 103-123

[29] Maldonato, N. M., Oliverio, A., Esposito, A. (2017) Neuronal Symphonies: Musical Improvisation and the Centrencephalic Space of Functional Integration, World Futures, pp. 1-20

[30] Maldonato, N. M., Dell'Orco, S., Esposito, A. (2016) The Emergence of Creativity. World Futures, 72(7-8), pp. 319-326

[31] Walton, A. E., Richardson, M. J., Langland-Hassan, P., Chemero, A. (2015) Improvisation and the Self-Organization of Multiple Musical Bodies, Frontiers in psychology, 6

[32] Mendonca, D. J., Al Wallace, W. (2007) A Cognitive Model of Improvisation in Emergency Management, IEEE Transactions on Systems, Man, and Cybernetics-Part A: Systems and Humans, 37(4), pp. 547-561

[33] Johnson-Laird, P. N. (2002) How Jazz Musicians Improvise, Music Perception: An Interdisciplinary Journal, 19(3), pp. 415-442

[34] Schmidt, R. A. (1976) Control Processes In Motor Skills, Exercise and Sport Sciences Reviews 4(1), pp. 229-261

[35] Kugler, P. N., Kelso, J. S., Turvey, M. (1980) On the Concept of Coordinative Structures as Dissipative Structures: I. Theoretical Lines of Convergence, Tutorials in Motor Behavior 3, pp. 3-47 
[36] Baer, J. (1996) The Effects of Task-Specific Divergent-Thinking Training, The Journal of Creative Behavior, 30(3), pp. 183-187

[37] Mullally, S. L., Maguire, E. A. (2014) Memory, Imagination, and Predicting the Future: a Common Brain Mechanism? The Neuroscientist 20(3), pp. 220-234

[38] Martin, A., Chao, L. L. (2001) Semantic Memory and the Brain: Structure and Processes, Current Opinion in Neurobiology, 11(2), pp. 194-201

[39] Beaty, R. E., Benedek, M., Wilkins, R. W., Jauk, E., Fink, A., Silvia, P. J., Neubauer, A. C. (2014) Creativity and the Default Network: A Functional Connectivity Analysis of the Creative Brain at Rest, Neuropsychologia 64, pp. $92-98$

[40] Sperandeo, R., Moretto, E., Baldo, G., Dell'Orco, S., \& Maldonato, N. M. (2017) Executive Functions and Personality Features: A Circular Interpretative Paradigm. In Cognitive Infocommunications (CogInfoCom), $20178^{\text {th }}$ IEEE International Conference on (pp. 000063-000066) IEEE

[41] Daitch, A. L., Sharma, M., Roland, J. L., Astafiev, S. V., Bundy, D. T., Gaona, C. M., Corbetta, M. (2013) Frequency-Specific Mechanism Links Human Brain Networks for Spatial Attention, Proceedings of the National Academy of Sciences, 110(48), pp. 19585-19590

[42] Engle, R. W. (2002) Working Memory Capacity as Executive Attention, Current Directions in Psychological Science, 11(1), pp. 19-23

[43] Schacter, D. L., Addis, D. R., Hassabis, D., Martin, V. C., Spreng, R. N., Szpunar, K. K. (2012) The Future of Memory: Remembering, Imagining, and the Brain, Neuron 76(4), pp. 677-694

[44] Bressler, S. L., Menon, V. (2010) Large-Scale Brain Networks in Cognition: Emerging Methods and Principles, Trends in Cognitive Sciences, 14(6), pp. 277-290

[45] Spreng, R. N., Andrews-Hanna, J. R. (2015) The Default Network and Social Cognition, Brain Mapping: an Encyclopedic Reference, 3, pp. 165169

[46] Christoff, K., Irving, Z. C., Fox, K. C., Spreng, R. N., Andrews-Hanna, J. R. (2016) Mind-Wandering as Spontaneous Thought: a Dynamic Framework, Nature Reviews Neuroscience, 17(11), pp. 718-731

[47] Corbetta, M. and Shulman, G. L. (2002) Control of Goal-directed and Stimulus-driven Attention in the Brain, Nature reviews neuroscience 3(3): 201-215

[48] Dreisbach, G. (2012) Mechanisms of Cognitive Control: The Functional Role of Task Rules, Current Directions in Psychological Science, 21(4), pp. 227-231 
[49] Goldman, A. (2013) Towards a Cognitive-Scientific Research Program for Improvisation: Theory and an Experiment, Psychomusicology: Music, Mind, and Brain 23(4), p. 210

[50] Dienes, Z. and Perner, J. (1999) A Theory of Implicit and Explicit Knowledge, Behavioral and Brain Sciences 22(05), pp. 735-808

[51] Schacter, D. L. (1987) Implicit Memory: History and Current Status, Journal of Experimental Psychology: Learning, Memory, and Cognition 13(3), pp. $501-518$

[52] Oliverio, A. (2008) Brain and Creativity, Progress of Theoretical Physics Supplement 173, pp. 66-78

[53] Graybiel, A. M. (1997) The Basal Ganglia and Cognitive Pattern Generators. Schizophrenia Bulletin 23(3), pp. 459-469

[54] Mele, A., Avena, M., Roullet, P., De Leonibus, E., Mandillo, S., Sargolini, F. and Oliverio, A. (2004) Nucleus Accumbens Dopamine Receptors in the Consolidation of Spatial Memory, Behavioural Pharmacology 15(5-6), pp. 423-431

[55] Cotterill, R. M. (2001) Cooperation of the Basal Ganglia, Cerebellum, Sensory Cerebrum and Hippocampus: Possible implications for cognition, Consciousness, Intelligence and Creativity, Progress in Neurobiology 64(1), pp. 1-33

[56] Boulez, P., Changeux, J. P. and Manoury, P. (2014) Les neurones enchantés: le cerveau et la musique. Odile Jacob

[57] Kutas, M., Hillyard, S. A. (1980) Reading Senseless Sentences: Brain Potentials Reflect Semantic Incongruity, Science, 207(4427), pp. 203-205

[58] Rowe, J. B., Eckstein, D., Braver, T., Owen, A. M. (2008) How does Reward Expectation Influence Cognition in the Human Brain? Journal of cognitive neuroscience 20(11), pp. 1980-1992

[59] Kane, M. J., Engle, R. W. (2002) The Role of Prefrontal Cortex in Working-Memory Capacity, Executive Attention, and General Fluid Intelligence: An Individual-Differences Perspective, Psychonomic Bulletin \& Review, 9(4), pp. 637-671

[60] Oken, B. S., Salinsky, M. C., Elsas, S. M. (2006) Vigilance, Alertness, or Sustained Attention: Physiological Basis and Measurement, Clinical Neurophysiology 117(9), pp. 1885-1901

[61] Fromm, E. (1978) Primary and Secondary Process in Waking and in Altered States of Consciousness, Journal of Altered States of Consciousness 4, pp. 115

[62] Glover, J. A., Ronning, R. R., Reynolds, C. (Eds.) (2013) Handbook of Creativity. Springer Science \& Business Media 
[63] Csikszentmihalyi, M. (1997) Flow and Creativity, Namta Journal, 22(2), pp. 60-97

[64] Dietrich, A. (2004) Neurocognitive Mechanisms Underlying the Experience of Flow, Consciousness and Cognition 13(4), pp. 746-761

[65] Ulrich, M., Keller, J., Hoenig, K., Waller, C. and Grön, G. (2014) Neural Correlates of Experimentally Induced Flow Experiences, Neuroimage 86, pp. 194-202

[66] Zahavi, D. (2003) Inner Time-Consciousness and Pre-Reflective SelfAwareness. The new Husserl: A critical reader, pp. 157-180

[67] Caan, W., Perrett, D. I. and Rolls, E. T. (1984) Responses of Striatal Neurons in the Behaving Monkey. 2. Visual processing in the caudal neostriatum, Brain research 290(1), pp. 53-65

[68] Báez-Mendoza, R., Schultz, W. (2013) The Role of the Striatum in Social Behavior, Frontiers in Neurosci. 7, pp. 233

[69] Thompson, R. F. (1986) The Neurobiology of Learning and Memory, Science, 233, pp. 941-948

[70] Brown, P., Marsden, C. D. (1998) What do the Basal Ganglia Do?. The Lancet, 351(9118), pp. 1801-1804

[71] Wei, D., Yang, J., Li, W., Wang, K., Zhang, Q., Qiu, J. (2014) Increased Resting Functional Connectivity of the Medial Prefrontal Cortex in Creativity by Means of Cognitive Stimulation, Cortex, 51, pp. 92-102

[72] Shamay-Tsoory, S. G., Adler, N., Aharon-Peretz, J., Perry, D., Mayseless, N. (2011) The Origins of Originality: the Neural Bases of Creative Thinking and Originality. Neuropsychologia 49(2), pp. 178-185

[73] Salimpoor, V. N., Zald, D. H., Zatorre, R. J., Dagher, A., McIntosh, A. R. (2015) Predictions and the Brain: How Musical Sounds Become Rewarding, Trends in Cognitive Sciences, 19(2), pp. 86-91

[74] Penfield, W. (1975) The Mysteries of the Mind. Princeton: Princeton University Press

[75] Koechlin, E., Hyafil, A. (2007) Anterior Prefrontal Function and the Limits of Human Decision-Making, Science, 318(5850), pp. 594-598

[76] Dehaene, S. (2014) Consciousness and the Brain: Deciphering How the Brain Codes Our Thoughts. Penguin

[77] Dehaene, S., Petit, C. (2009) Parole et musique: aux origines du dialogue humain. Odile Jacob 